\title{
micromachines
}

ISSN 2072-666X

www.mdpi.com/journal/micromachines

Article

\section{Effects of Fumed and Mesoporous Silica Nanoparticles on the Properties of Sylgard 184 Polydimethylsiloxane}

\author{
Junshan Liu ${ }^{1,2, *}$, Guoge Zong ${ }^{1}$, Licheng He ${ }^{1}$, Yangyang Zhang ${ }^{1}$, Chong Liu ${ }^{1}$ and Liding Wang ${ }^{1}$ \\ 1 Key Laboratory for Micro/Nano Technology and System of Liaoning Province, Dalian University \\ of Technology, Dalian 116024, China; E-Mails: zongguoge@163.com (G.Z.); \\ helicheng868@163.com (L.H.); 15104504983@163.com (Y.Z); chong1@dlut.edu.cn (C.L.); \\ wangld@dlut.edu.cn (L.W.) \\ 2 Key Laboratory for Precision and Non-traditional Machining Technology of Ministry of Education, \\ Dalian University of Technology, Dalian 116024, China \\ * Author to whom correspondence should be addressed; E-Mail: liujs@dlut.edu.cn; \\ Tel.: +86-411-8470-7713 (ext. 2171); Fax: +86-411-8470-7940.
}

Academic Editor: Hongrui Jiang

Received: 21 April 2015 / Accepted: 2 July 2015 / Published: 8 July 2015

\begin{abstract}
The effects of silica nanoparticles on the properties of a commonly used Sylgard 184 polydimethylsiloxane (PDMS) in microfluidics were systemically studied. Two kinds of silica nanoparticles, A380 fumed silica nanoparticles and MCM-41 mesoporous silica nanoparticles, were individually doped into PDMS, and the properties of PDMS with these two different silica nanoparticles were separately tested and compared. The thermal and mechanical stabilities of PDMS were significantly enhanced, and the swelling characteristics were also improved by doping these two kinds of nanoparticles. However, the transparency of PDMS was decreased due to the light scattering by nanoparticles. By contrast, PDMS/MCM-41 nanocomposites showed a lower coefficient of thermal expansion (CTE) owing to the mesoporous structure of MCM-41 nanoparticles, while PDMS/A380 nanocomposites showed a larger elastic modulus and better transparency due to the smaller size of A380 nanoparticles. In addition, A380 and MCM-41 nanoparticles had the similar effects on the swelling characteristics of PDMS. The swelling ratio of PDMS in toluene was decreased to 0.68 when the concentration of nanoparticles was $10 \mathrm{wt} \%$.
\end{abstract}

Keywords: polydimethylsiloxane; PDMS; Sylgard 184; silica; nanoparticles 


\section{Introduction}

Polydimethylsiloxane (PDMS) has been widely used in microfluidics due to its flexibility, biocompatibility, transparency, low cost and ease of fabrication [1-7]. However, PDMS has a high coefficient of thermal expansion (CTE) and a low elastic modulus, which makes its poor thermal and mechanical stabilities and hinders many practical applications. For example, the metal microelectrode has become one of the essential compositions of microfluidic chips. However, wrinkles or even cracks were caused in metal films deposited on PDMS substrates owing to poor stabilities of PDMS [7-9]. In addition to stabilities, swelling of PDMS is another important issue [10-12]. The swelling ratios of PDMS are very large in common organic solvents, such as a swelling ratio of 1.15 in toluene [10]. Hence, it is difficult to perform experiments in organic media for PDMS microfluidic chips.

To improve the stabilities of PDMS, many kinds of nano materials, such as carbon nanotubes (CNTs) [13-15], silica nanoparticles [16-18], and graphene [19] have been doped into PDMS. Wu et al. reported that the elastic modulus of PDMS was increased to $2.34 \mathrm{MPa}$ by adding carbon nanotubes [13]. Camenzind et al. reported that the elastic modulus of PDMS was increased to $3.18 \mathrm{MPa}$ by admixing fumed silica nanoparticles [16]. Chen et al. reported a method for embedding CNT sheets in PDMS, and the CTE of PDMS was reduced to $6 \mathrm{ppm} /{ }^{\circ} \mathrm{C}$ in the direction parallel to CNT alignment [15]. Yamauchi's group has investigated thermal, mechanical, optical and swelling properties of PDMS/silica nanocomposites [17,18]. The mechanical and thermal stabilities were effectively enhanced, and the swelling characteristics of PDMS were also improved. However, in their studies, a hard PDMS (X-32-3095, Shin-Etsu Chemical Co., Ltd, Tokyo, Japan), which does not require a curing agent, was used.

In this paper, we systematically studied the effects of silica nanoparticles on the properties of the most commonly used PDMS in microfluidics, Sylgard 184 (Dow Corning Corporation, Midland, MI, USA) $[3,5,10,11,20]$. The thermal and mechanical stabilities, swelling characteristics and transparency were all examined. Two kinds of silica nanoparticles, fumed silica nanoparticles and mesoporous silica nanoparticles were doped and compared.

\section{Experimental Section}

\subsection{Materials}

A380 fumed silica nanoparticles were purchased from Evonik Degussa (Essen, Germany), and MCM-41 mesoporous silica nanoparticles were provided by Nanjing XFNANO Materials (Nanjing, China). These two types of nanoparticles are abbreviated henceforward as A380 and MCM-41, respectively. Specific characteristics of these two types of silica nanoparticles are listed in Table 1. According to the density of silica $\left(2.2 \mathrm{~g} / \mathrm{cm}^{3}\right)$ and the particle size and pore volume of silica nanoparticles listed in Table 1, the mass of a single nanoparticle was approximately calculated. A single A380 nanoparticle was about $4 \times 10^{-19} \mathrm{~g}$, and a single MCM-41 nanoparticle was about $1 \times 10^{-14} \mathrm{~g}$.

PDMS (Sylgard 184) consisted of a base and a curing agent was purchased from Dow Corning Corporation. 
Table 1. Specific characteristics of silica nanoparticles (SSA, specific surface area).

\begin{tabular}{ccccc}
\hline The Type & SSA $\left(\mathbf{m}^{2} / \mathbf{g}\right)$ & Particle size $(\mathbf{n m})$ & Pore diameter $(\mathbf{n m})$ & Pore volume $\left(\mathrm{cm}^{3} / \mathbf{g}\right)$ \\
\hline A380 & $\sim 380$ & $\sim 7$ & - & - \\
MCM-41 & $\sim 800$ & $\sim 300$ & $3.5-4$ & $0.8-0.9$ \\
\hline
\end{tabular}

\subsection{Fabrication of PDMS/Silica Nanocomposites}

During the process of fabricating PDMS/silica nanocomposites, the agglomeration of nanoparticles is inevitable. In order to obviate the agglomeration and achieve a homogeneous dispersion, magnetic stirring and sonication were often used [13,19]. In addition, organic solvents were often used to help the dispersion of nanoparticles, such as toluene [21,22] and xylene [13,19]. Our group once used toluene to dilute PDMS for making a thin film [23], hence, here, toluene was also chosen as the dilute solvent. In this study, PDMS/silica nanocomposites were fabricated by combining magnetic stirring, sonication and the dilute solvent. The whole fabrication procedures were illustrated in Figure 1: (a) Silica nanoparticles were mixed with toluene. The mixture was magnetically stirred for $1 \mathrm{~h}$, and ultrasonically oscillated for $3 \mathrm{~h}$. (b) The nanoparticles in toluene were mixed with the PDMS base. Similarly, the mixture was stirred for $1 \mathrm{~h}$ and oscillated for $3 \mathrm{~h}$. (c) Toluene in the mixture was thoroughly evaporated in chemical hood. (d) The PDMS base and curing agent were mixed at a weight ratio of 10:1. The mixture was stirred uniformly, and then put into a vacuum oven to remove bubbles. (e) The mixture was poured on a glass plate, and cured at $80^{\circ} \mathrm{C}$ for $2 \mathrm{~h}$. Then the PDMS/silica nanocomposites were peeled off from the glass plate.

\subsection{Characterization}

Silica nanoparticles were observed by a transmission electron microscope (TEM) (Tecnai G2 F30, FEI Company, Hillsboro, OR, USA), as shown in Figure 2. The thermal expansion property of PDMS/silica nanocomposites was examined by a thermomechanical analyzer (Q400, TA Instruments, New Castle, DE, USA). A specimen with a dimension of $8 \mathrm{~mm} \times 5 \mathrm{~mm} \times 5 \mathrm{~mm}$ was prepared. During the test, the temperature was increased from room temperature to $180{ }^{\circ} \mathrm{C}$ at a rate of $5{ }^{\circ} \mathrm{C} / \mathrm{min}$. The CTE of PDMS/silica nanocomposites was determined based on the curves from the analyzer. The elastic modulus of PDMS/silica nanocomposites was measured by using a universal tensile testing machine (5657, Instron Company, Norwood, MA, USA). The specimen was fabricated according to American Society for Testing of Materials (ASTM) standards, and the rate of crosshead motion was kept at $50 \mathrm{~mm} / \mathrm{min}$. To measure the swelling property of PDMS/silica nanocomposites, a specimen with a dimension of $25 \mathrm{~mm} \times 25 \mathrm{~mm} \times 2 \mathrm{~mm}$ was put into toluene, and the weight of the specimen was recorded every half an hour until the swelling of nanocomposites reached equilibrium. In accordance with the change in weight caused by absorption, the swelling ratio was calculated [10]. The transmittance of PDMS/silica nanocomposites was measured by a UV-Vis spectrophotometer (Cary300, Agilent Technologies, Santa Clara, CA, USA), and the thickness of the specimen was $100 \mu \mathrm{m}$. 
(a)

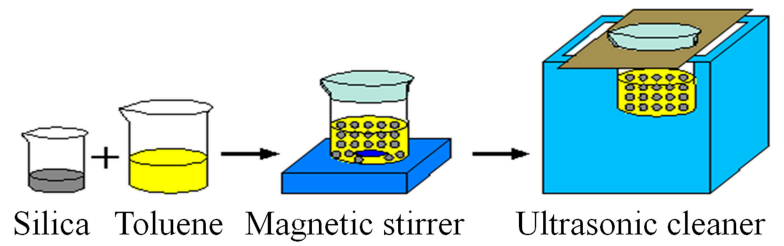

(b)

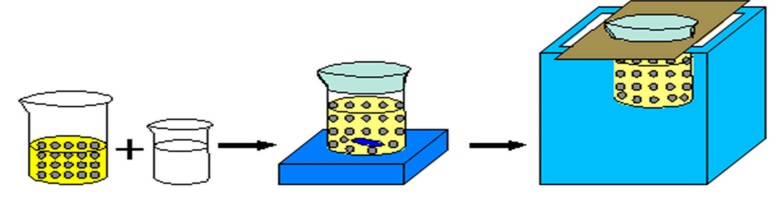

PDMS base

(c)

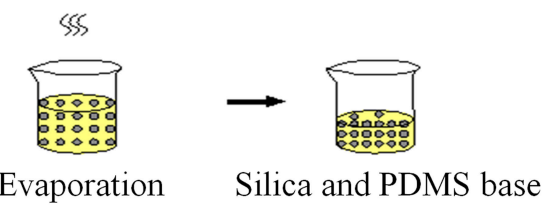

(d)

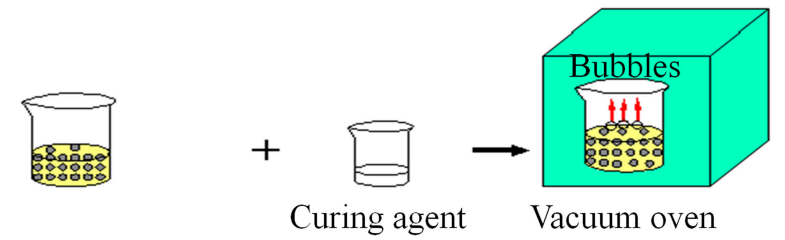

(e)

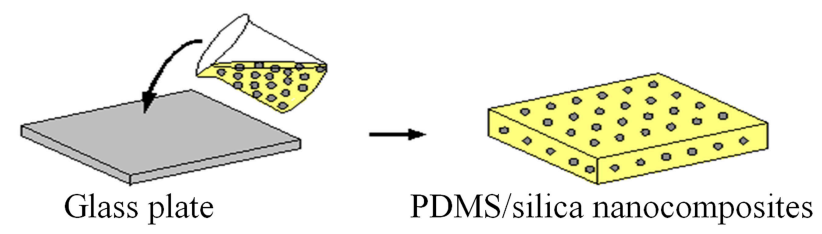

Figure 1. Fabrication procedures of PDMS/silica nanocomposites. (a) Dispersion of silica nanoparticles in toluene under magnetic stirring and ultrasonic vibration; (b) mixing silica nanoparticles with PDMS base under magnetic stirring and ultrasonic vibration; (c) evaporation of toluene; (d) mixing PDMS base with curing agent and removing bubbles; and (e) cast molding of PDMS/silica nanocomposites on a glass plate.
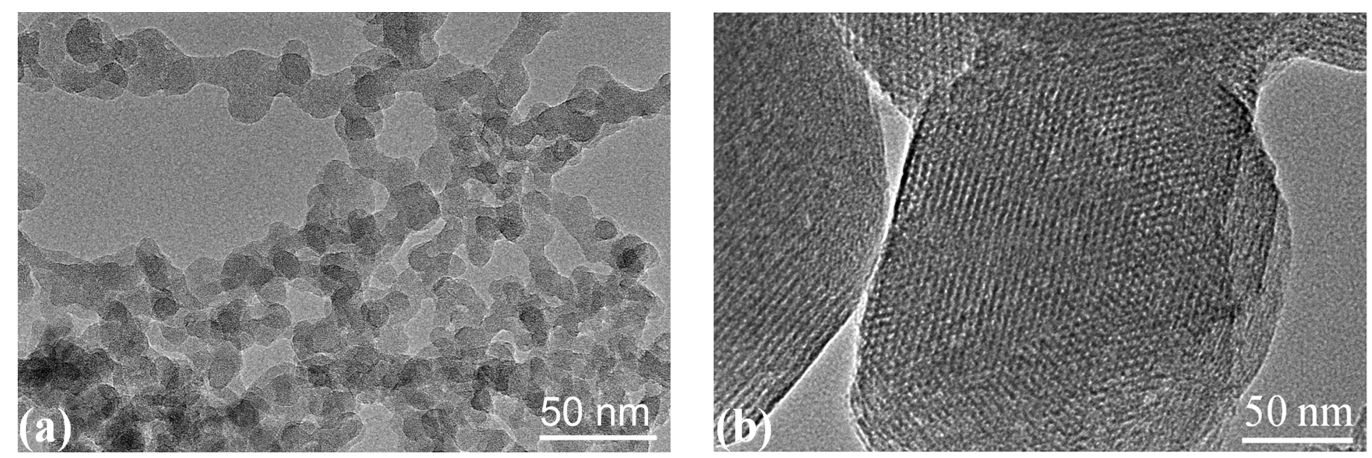

Figure 2. Transmission electron microscope (TEM) images of silica nanoparticles. (a) A380 fumed silica nanoparticles; (b) MCM-41 mesoporous silica nanoparticles.

\section{Results and Discussion}

It is worth noting that the Sylgard 184 PDMS base itself contains silica filler. However, these silica filler are dimethylvinylated and trimethylated silica [24], and different from the silica nanoparticles (A380 and MCM-41) used in this study. Moreover, during the curing process, the vinyl of the silica 
filler and the silicon hydride groups of the curing agent would undergo a hydrosilylation reaction to form a Si-C bond [24]. Therefore, it is believed that the silica nanoparticles used in this work would not interact with the silica filler, but with the whole three-dimensional PDMS network.

\subsection{The CTE of PDMS/Silica Nanocomposites}

Strain-temperature curves of PDMS/silica nanocomposites were shown in Figure 3, and the CTE of PDMS/silica nanocomposites was calculated from the slope of the curves. The CTE of pure PDMS was $301 \mathrm{ppm} /{ }^{\circ} \mathrm{C}$. The CTE of PDMS decreased with the silica concentration. The decrease of the CTE was mainly attributed to the following two factors. On one hand, the CTE of silica is only $0.54 \mathrm{ppm} /{ }^{\circ} \mathrm{C}$ [25], which is almost four orders of magnitude lower than that of pure PDMS. Hence the CTE of PDMS can be decreased by adding silica nanoparticles. On the other hand, covalent bonds between silica nanoparticles and PDMS and hydrogen bonds between silica nanoparticles formed [16,26]. The bonds caused large interaction between PDMS and silica nanoparticles, and the interaction restricted the heat deformation of PDMS.
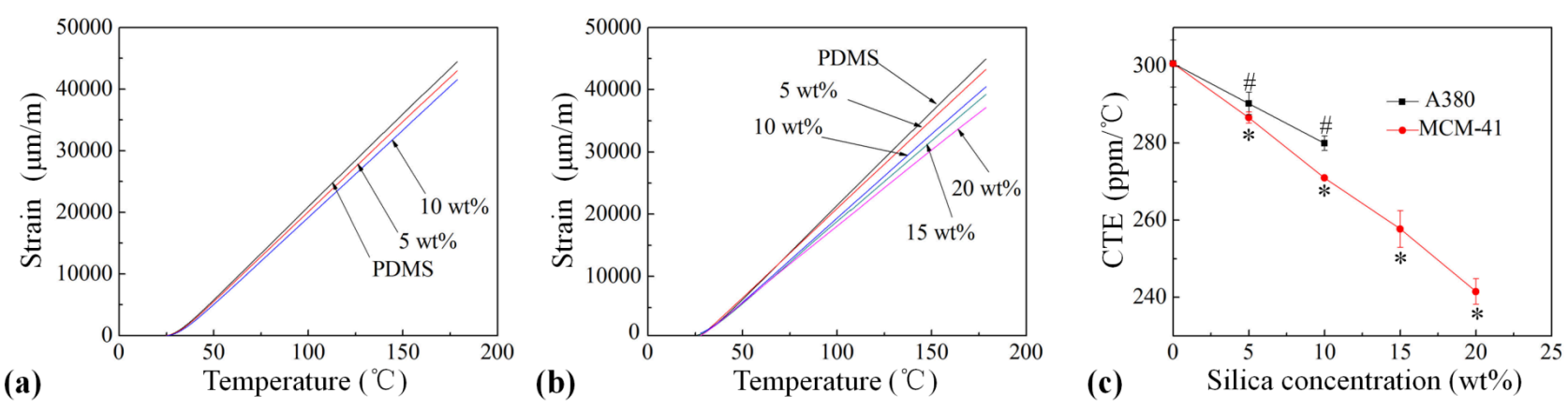

Figure 3. Strain-temperature curves of PDMS with different concentrations of (a) A380 fumed silica nanoparticles and (b) MCM-41 mesoporous silica nanoparticles; (c) CTEs of PDMS/A380 and PDMS/MCM-41 nanocomposites. Data were shown as mean \pm standard deviation (SD). \#, $p<0.05$, compared with control of PDMS/A380 nanocomposites; $*, p<0.05$, compared with control of PDMS/MCM-41 nanocomposites (one-way analysis of variance, Tukey's post hoc test).

Compared with A380, MCM-41 reduced the CTE of PDMS more efficiently. At a concentration of $10 \mathrm{wt} \%$, the CTE of PDMS/MCM-41 nanocomposites was $272 \mathrm{ppm} /{ }^{\circ} \mathrm{C}$, while that of PDMS/A380 nanocomposites was $280 \mathrm{ppm} /{ }^{\circ} \mathrm{C}$. The width of the PDMS chain is about $0.7 \mathrm{~nm}$ [27], and the pore diameter of MCM-41 is 3.5-4 nm (Table 1). A portion of PDMS chains could be easily penetrated into the pores of MCM-41 due to capillary force [28]. According to the research by Yamauchi's group [17,18], the thermal expansion of these PDMS chains confined inside the pores of MCM-41 was restricted by the framework of MCM-41. These confined PDMS chains made few contributions to the CTE of PDMS. Therefore, PDMS/MCM-41 nanocomposites showed a lower CTE at the same concentration of silica nanoparticles.

Moreover, it was observed that the viscosity of the mixture of PDMS and A380 was apparently larger than that of the mixture of PDMS and MCM-41. At the same concentration of nanoparticles, due to a smaller size, the number of A380 is about 4 orders of magnitude larger than that of MCM-41, and 
the distance among A380 is also smaller. Therefore, we believed that the interaction among A380 could be stronger, and the relative motion among A380 could be more difficult, so the viscosity of the mixture of PDMS and A380 was larger. It became very difficult to mix PDMS base and A380 when the concentration of A380 was over $10 \mathrm{wt} \%$. While the concentration of MCM-41 was easily increased up to $20 \mathrm{wt} \%$, and, correspondingly, the CTE of PDMS was decreased to $241 \mathrm{ppm} /{ }^{\circ} \mathrm{C}$.

\subsection{The Elastic Modulus of PDMS/Silica Nanocomposites}

The elastic modulus $(E)$ of PDMS/silica nanocomposites was calculated based on the stress-strain region below 40\%. Stress-strain curves of PDMS are shown in Figure 4a,b, and effects of concentrations of nanoparticles on the modulus are shown in Figure 4c. The elastic modulus of pure PDMS was $1.38 \mathrm{MPa}$. It can be seen that silica nanoparticles significantly improved the elastic modulus of PDMS. The modulus of PDMS with $20 \mathrm{wt} \% \mathrm{MCM}-41$ was $9.44 \mathrm{MPa}$, which is almost seven times of that of pure PDMS. The increase of the elastic modulus mainly resulted from two aspects. First, the elastic modulus of silica is at least four orders of magnitude larger than that of PDMS, and, therefore, the modulus of PDMS increased with the amount of nanoparticles. Second, hydrogen bonds between silica nanoparticles and covalent bonds between silica and PDMS increased the resistance to deformation [29].

In addition, the elastic modulus of PDMS/A380 nanocomposites was apparently larger than that of PDMS/MCM-41 nanocomposites at the same concentration. At a concentration of $10 \mathrm{wt} \%$, the elastic modulus of PDMS/A380 nanocomposites was $7.83 \mathrm{MPa}$, while that of PDMS/MCM-41 nanocomposites was only $5.44 \mathrm{MPa}$. As stated above, the interaction among A380 and between A380 and PDMS could be stronger due to the smaller size of A380, and the stronger interaction made a larger elastic modulus.
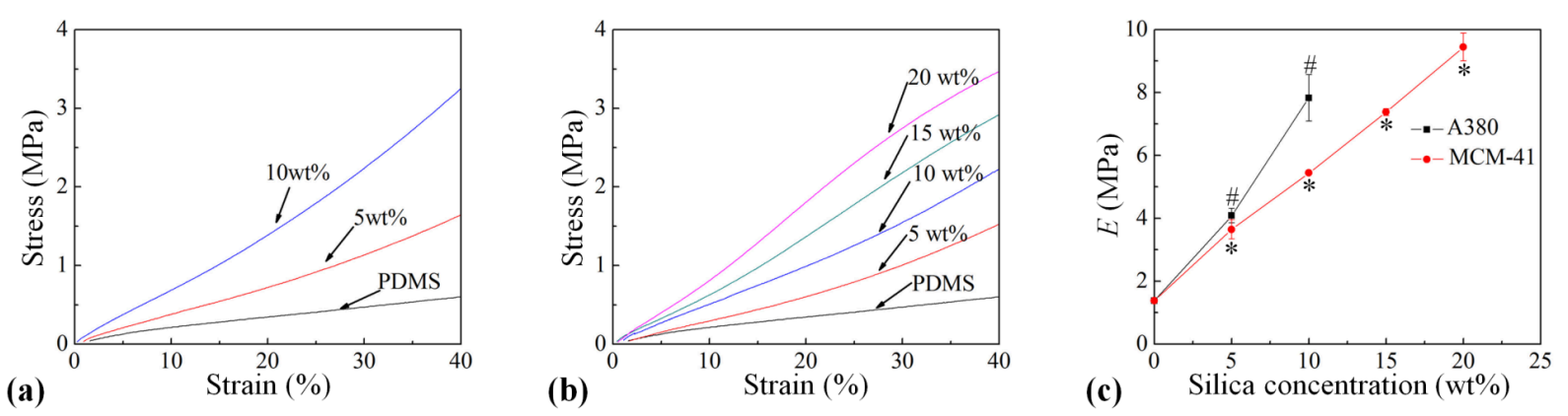

Figure 4. Stress-strain curves of PDMS with different concentrations of (a) A380 fumed silica nanoparticles and (b) MCM-41 mesoporous silica nanoparticles; (c) Elastic moduli of PDMS/A380 and PDMS/MCM-41 nanocomposites. Data were shown as mean \pm standard deviation (SD). \#, $p<0.05$, compared with control of PDMS/A380 nanocomposites; $*, p<0.05$, compared with control of PDMS/MCM-41 nanocomposites (one-way analysis of variance, Tukey's post hoc test).

\subsection{Swelling of PDMS/Silica Nanocomposites}

The swelling property of PDMS/silica nanocomposites in toluene was examined. The swelling ratio $(R)$ was calculated as $\left(W-W_{0}\right) / W_{0}$ where $W_{0}$ and $W$ denoted weights of the PDMS before and after 
absorbing toluene. As shown in Figure 5, at the beginning, the swelling ratio of PDMS increased quickly, and reached the maximum after about $2 \mathrm{~h}$. Then the ratio kept nearly constant with time. The swelling ratio of pure PDMS was 1.142. The ratio remarkably decreased with the silica concentration, and the ratio was even down to 0.486 when the concentration of MCM-41 was $20 \mathrm{wt} \%$, which is almost $60 \%$ lower than that of pure PDMS. The silica nanoparticles increased the crosslinking density of PDMS [26]. According to the Flory-Rehner rubber swelling theory [30], the increase of crosslinking density decreased the swelling ratio of PDMS.

Compared with MCM-41, the reduction of the swelling ratio caused by A380 was expected to be more effective because the crosslinking density of PDMS/A380 nanocomposites was denser due to its smaller size [16]. However, as shown in Figure 5, the swelling ratios of PDMS/A380 and PDMS/MCM-41 nanocomposites were very close at the same concentration. For example, at the concentration of $10 \mathrm{wt} \%$, the swelling ratio of PDMS/A380 nanocomposites was 0.681 , and that of PDMS/MCM-41 nanocomposites as 0.686. As mentioned above, a portion of PDMS was confined inside the nanopores in MCM-41. We consider that the absorbing ability of this portion of PDMS was suppressed by the frameworks of MCM-41, which led to an average smaller swelling ratio of the whole PDMS.

(a)

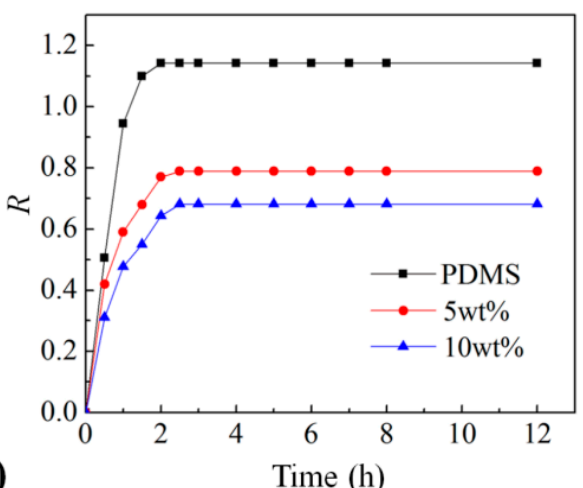

(b)

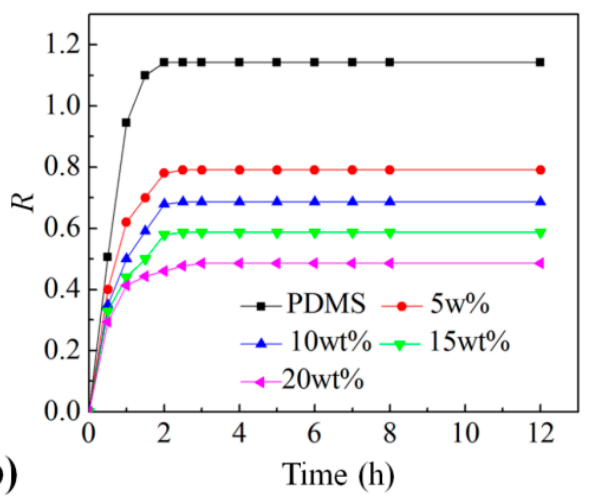

Figure 5. Time-dependent swelling ratios of PDMS with different concentrations of (a) A380 fumed silica nanoparticles and (b) MCM-41 mesoporous silica nanoparticles in toluene.

\subsection{Transparency of PDMS/Silica Nanocomposites}

The effect of silica nanoparticles on the transparency of PDMS was studied. The ultraviolet-visible spectroscopy (UV-Vis) spectra of PDMS/silica nanocomposites are shown in Figure 6. The pure PDMS had excellent transparency, and the transmittance was above $95 \%$ in the range from 350 to $800 \mathrm{~nm}$. The silica nanoparticles can cause significant light scattering, and this will decrease the transmittance of PDMS. As shown in Figure 6, the transmittance of PDMS decreased with the concentration of nanoparticles. Moreover, the smaller the wavelength was, the lower the transmittance of PDMS/silica nanocomposites was.

Furthermore, PDMS/A380 nanocomposites showed much better transparency than PDMS/MCM-41 nanocomposites at the same concentration. At the concentration of $10 \mathrm{wt} \%$, the transmittance of PDMS/A380 nanocomposites was $81 \%$ at $450 \mathrm{~nm}$, while that of PDMS/MCM-41 nanocomposites was $67 \%$ at $450 \mathrm{~nm}$. The transmittance of PDMS/silica nanocomposites is mainly related to the size and 
amount of nanoparticles [31]. Hence, when the concentration of nanoparticles is the same, the transmittance is dependent on the size of nanoparticles. As shown in Table 1, the size of A380 is much smaller than MCM-41. Therefore, the scattering loss caused by A380 is smaller, and a better transparency of PDMS/A380 nanocomposites can be obtained.
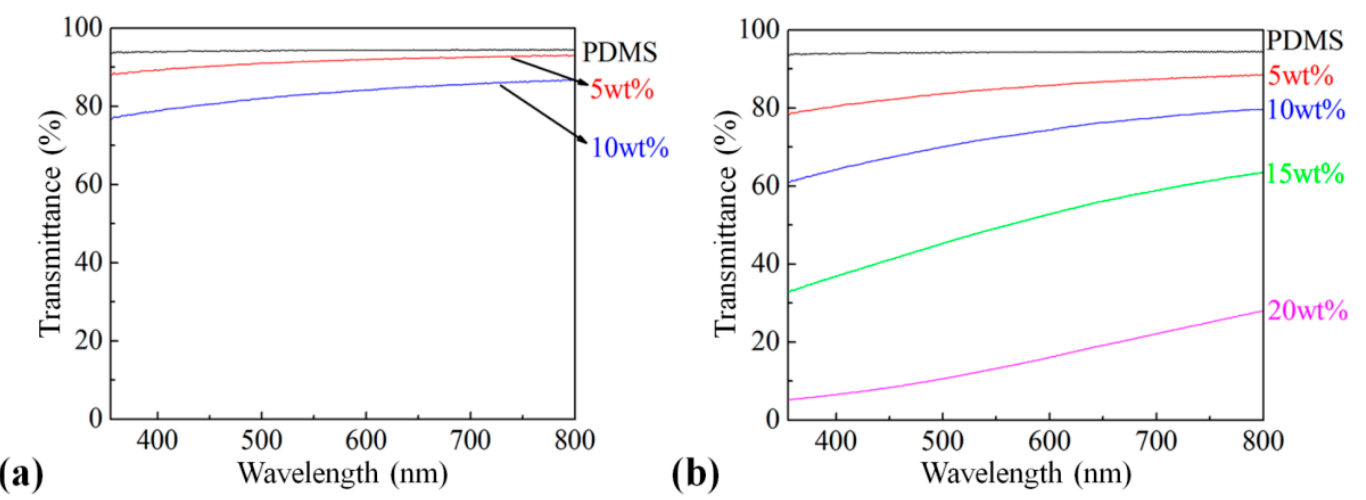

Figure 6. Ultraviolet-visible spectroscopy (UV-Vis) spectra of PDMS with different concentrations of (a) A380 fumed silica nanoparticles and (b) MCM-41 mesoporous silica nanoparticles.

\section{Conclusions}

The effects of two kinds of silica nanoparticles on the CTE, elastic modulus, swelling property and transparency of Sylgard 184 PDMS were investigated and compared. By doping silica nanoparticles, the stabilities and swelling characteristics of PDMS were remarkably improved, while the transmittance was decreased. Compared with A380, the reduction of CTE of PDMS caused by MCM-41 was more effective due to its mesoporous structure. Moreover, a higher concentration of MCM-41 could be doped owing to its larger size, and a CTE of $241 \mathrm{ppm} /{ }^{\circ} \mathrm{C}$ was obtained at a concentration of $20 \mathrm{wt} \%$. Owing to the smaller size of A380, the PDMS/A380 nanocomposites showed larger elastic moduli and better transparency than PDMS/MCM-41 nanocomposites. A380 and MCM-41 nanoparticles had the similar effects on the swelling property of PDMS. In sum, by doping different types of silica nanoparticles, Sylgard 184 PDMS with different properties can be achieved and applied for a variety of applications.

\section{Acknowledgments}

This work was supported by the National Natural Science Foundation of China (51475080, 51321004), the National High-tech R\&D Program of China (2012AA040406), the Fundamental Research Funds for the Central Universities (DUT14QY21), Dalian Foundation of Science and Technology (2012J21DW002), and Funds of Key Laboratory of Liaoning Education Department (LZ2014005).

\section{Author Contributions}

Junshan Liu, Chong Liu and Liding Wang conceived and designed the experiments; Guoge Zong, Licheng He and Yangyang Zhang performed the experiments; Junshan Liu and Guoge Zong analyzed the data; Junshan Liu and Guoge Zong wrote the paper. 


\section{Conflicts of Interest}

The authors declare no conflict of interest.

\section{References}

1. Xia, Y.N.; Whitesides, G.M. Soft lithography. Angew. Chem. Int. Ed. 1998, 37, 550-575.

2. McDonald, J.C.; Whitesides, G.M. Poly(dimethylsiloxane) as a material for fabricating microfluidic devices. Accounts Chem. Res. 2002, 35, 491-499.

3. Bonk, S.M.; Oldorf, P.; Peters, R.; Baumann, W.; Gimsa, J. Fast prototyping of sensorized cell culture chips and microfluidic systems with ultrashort laser pulses. Micromachines 2015, 6, 364-374.

4. Pinto, E.; Faustino, V.; Rodrigues, R.O.; Pinho, D.; Garcia, V.; Miranda, J.M.; Lima, R. A rapid and low-cost nonlithographic method to fabricate biomedical microdevices for blood flow analysis. Micromachines 2015, 6, 121-135.

5. Yu, Z.; Amirouche, F. An electromagnetically-actuated all-PDMS valveless micropump for drug delivery. Micromachines 2011, 2, 345-355.

6. Wu, W.Y.; Zhong, X.; Wang, W.; Miao, Q.A.; Zhu, J.J. Flexible PDMS-based three-electrode sensor. Electrochem. Commun. 2010, 12, 1600-1604.

7. Guo, L.; DeWeerth, S.P. An effective lift-off method for patterning high-density gold interconnects on an elastomeric substrate. Small 2010, 6, 2847-2852.

8. Graudejus, O.; Goerrn, P.; Wagner, S. Controlling the morphology of gold films on poly(dimethylsiloxane). ACS Appl. Mater. Interfaces 2010, 2, 1927-1933.

9. Bowden, N.; Brittain, S.; Evans, A.G.; Hutchinson, J.W.; Whitesides, G.M. Spontaneous formation of ordered structures in thin films of metals supported on an elastomeric polymer. Nature 1998, 393, 146-149.

10. Kim, B.Y.; Hong, L.Y.; Chung, Y.M.; Kim, D.P.; Lee, C.S. Solvent-resistant PDMS microfluidic devices with hybrid inorganic/organic polymer coatings. Adv. Funct. Mater. 2009, 19, 3796-3803.

11. Lee, J.N.; Park, C.; Whitesides, G.M. Solvent compatibility of poly(dimethylsiloxane)-based microfluidic devices. Anal. Chem. 2003, 75, 6544-6554.

12. Koh, K.S.; Chin, J.; Chia, J.; Chiang, C.L. Quantitative studies on PDMS-PDMS interface bonding with piranha solution and its swelling effect. Micromachines 2012, 3, 427-441.

13. Wu, C.L.; Lin, H.C.; Hsu, J.S.; Yip, M.C.; Fang, W. Static and dynamic mechanical properties of polydimethylsiloxane/carbon nanotube nanocomposites. Thin Solid Films 2009, 517, 4895-4901.

14. Vast, L.; Carpentier, L.; Lallemand, F.; Colomer, J.F.; van Tendeloo, G.; Fonseca, A.; Delhalle, J. Multiwalled carbon nanotubes functionalized with 7-octenyltrichlorosilane and n-octyltrichlorosilane: Dispersion in Sylgard ${ }^{\circledR} 184$ silicone and Young's modulus. J. Mater. Sci. 2009, 44, 3476-3482.

15. Chen, L.; Liu, C.; Liu, K.; Meng, C.; Hu, C.; Wang, J.; Fan, S. High-performance, low-voltage, and easy-operable bending actuator based on aligned carbon nanotube/polymer composites. ACS Nano 2011, 5, 1588-1593.

16. Camenzind, A.; Schweizer, T.; Sztucki, M.; Pratsinis, S.E. Structure \& strength of silica-PDMS nanocomposites. Polymer 2010, 51, 1796-1804. 
17. Suzuki, N.; Kiba, S.; Kamachi, Y.; Miyamoto, N.; Yamauchi, Y. Unusual reinforcement of silicone rubber compounds containing mesoporous silica particles as inorganic fillers. Phys. Chem. Chem. Phys. 2012, 14, 3400-3407.

18. Suzuki, N.; Kamachi, Y.; Takai, K.; Kiba, S.; Sakka, Y.; Miyamoto, N.; Yamauchi, Y. Effective use of mesoporous silica filler: Comparative study on thermal stability and transparency of silicone rubbers loaded with various kinds of silica particles. Eur. J. Inorg. Chem. 2014, 2014, 2773-2778.

19. Ozbas, B.; O’Neill, C.D.; Register, R.A. Multifunctional elastomer nanocomposites with functionalized graphene single sheets. J. Polym. Sci. B Polym. Phys. 2012, 50, 910-916.

20. Johnston, I.D.; McCluskey, D.K.; Tan, C.K.L.; Tracey, M.C. Mechanical characterization of bulk Sylgard 184 for microfluidics and microengineering. J. Micromech. Microeng. 2014, 24, 035017.

21. Liu, C.X.; Choi, J.W. Patterning conductive PDMS nanocomposite in an elastomer using microcontact printing. J. Micromech. Microeng. 2009, 19, 085019.

22. Kong, K.T.S.; Mariatti, M.; Rashid, A.A.; Busfield, J.J.C. Enhanced conductivity behavior of polydimethylsiloxane (PDMS) hybrid composites containing exfoliated graphite nanoplatelets and carbon nanotubes. Compos. Part B Eng. 2014, 58, 457-462.

23. Liu, J.; Xu, F.; Wang, S.; Chen, Z.; Pan, J.; Ma, X.; Jiao, X.; Xu, Z.; Liu, C.; Wang, L. A polydimethylsiloxane electrophoresis microchip with a thickness controllable insulating layer for capacitatively coupled contactless conductivity detection. Electrochem. Commun. 2012, 25, 147-150.

24. Lee, J.N.; Jiang, X.; Ryan, D.; Whitesides, G.M. Compatibility of mammalian cells on surfaces of poly(dimethylsiloxane). Langmuir 2004, 20, 11684-11691.

25. Duwe, S.; Arlt, C.; Aranda, S. A detailed thermal analysis of nanocomposites filled with $\mathrm{SiO}_{2}$, AlN or boehmite at varied contents and a review of selected rules of mixture. Compos. Sci. Technol. 2012, 72, 1324-1330.

26. Kaneko, M.L.Q.A.; Yoshida, I.V.P. Effect of natural and organically modified montmorillonite clays on the properties of polydimethylsiloxane rubber. J. Appl. Polym. Sci. 2008, 108, 2587-2596.

27. Horn, R.G.; Hirz, S.J.; Hadziioannou, G.; Frank, C.W.; Catala, J.M. A reevaluation of forces measured across thin polymer films: Nonequilibrium and pinning effects. J. Chem. Phys. 1989, 90, 6767-6774.

28. Suzuki, N.; Kiba, S.; Yamauchi, Y. Bimodal filler system consisting of mesoporous silica particles and silica nanoparticles toward efficient suppression of thermal expansion in silica/epoxy composites. J. Mater. Chem. 2011, 21, 14941-14947.

29. Shim, S.E.; Isayev, A.I. Rheology and structure of precipitated silica and poly(dimethyl siloxane) system. Rheol. Acta 2004, 43, 127-136.

30. Flory, P.J.; Rehner, J., Jr. Statistical mechanics of cross-linked polymer networks II. Swelling. J. Chem. Phys. 1943, 11, 521-526.

31. Novak, B.M. Hybrid nanocomposite materials-between inorganic glasses and organic polymers. Adv. Mater. 1993, 5, 422-433.

(C) 2015 by the authors; licensee MDPI, Basel, Switzerland. This article is an open access article distributed under the terms and conditions of the Creative Commons Attribution license (http://creativecommons.org/licenses/by/4.0/). 